INPLASY

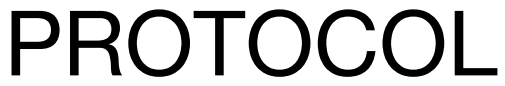

To cite: Mahmoudi et al.

Application of digital health for improving medication adherence in MS patients. Inplasy protocol 2021100058. doi:

10.37766/inplasy2021.10.0058

Received: 17 October 2021

Published: 17 October 2021

Corresponding author:

Farhad Mahmoudi

farhad.mg116@yahoo.com

Author Affiliation:

Isfahan University of Medical

Sciences, Isfahan, Iran

Support: None.

Review Stage at time of this submission: Data extraction.

Conflicts of interest:

None declared.

\section{Application of digital health for improving medication adherence in MS patients}

Mahmoudi, F1; Mokarram, M2; Saboudi, S3; Hashemi, S4; Saberi, P5; Zamanian, $\mathrm{H}^{6}$.

Review question / Objective: The aim of this study is to evaluate the efficacy of digital health interventions in monitoring and improving medication adherence in Multiple Sclerosis patients.

Condition being studied: Multiple sclerosis (MS) is the most prevalent chronic inflammatory disease of the central nervous system (CNS), which leads to focal lesions in the white matter, characterized by selective primary demyelination with partial preservation of axons and reactive astrocytic gliosis. The disease is thought to be due to a complex interaction between different genetic and environmental factors. The prevalence of MS is rising all over the world, due on one hand to earlier diagnosis and prolonged survival, and on the other to a true increase in incidence of the disease. The diagnosis of MS remains clinical despite recent advances in diagnostics and relies on demonstrating dissemination in space and time while excluding alternative diagnoses.

INPLASY registration number: This protocol was registered with the International Platform of Registered Systematic Review and Meta-Analysis Protocols (INPLASY) on 17 October 2021 and was last updated on 17 October 2021 (registration number INPLASY2021100058).

\section{INTRODUCTION}

Review question / Objective: The aim of this study is to evaluate the efficacy of digital health interventions in monitoring and improving medication adherence in Multiple Sclerosis patients
Condition being studied: Multiple sclerosis (MS) is the most prevalent chronic inflammatory disease of the central nervous system (CNS), which leads to focal lesions in the white matter, characterized by selective primary demyelination with partial preservation of axons and reactive astrocytic gliosis. The disease is thought to 
be due to a complex interaction between different genetic and environmental factors. The prevalence of MS is rising all over the world, due on one hand to earlier diagnosis and prolonged survival, and on the other to a true increase in incidence of the disease. The diagnosis of MS remains clinical despite recent advances in diagnostics and relies on demonstrating dissemination in space and time while excluding alternative diagnoses.

\section{METHODS}

Search strategy: We searched different databases, our search string for PUBMED is: (digital*[tiab] OR telemedicine[tiab] OR tele*[tiab] OR mobile[tiab] OR mobile phone[tiab]OR "Mobile App"[ti] OR "Mobile Appl*"[ti] OR "Mobile Apps"[ti] OR mhealth OR "m-health"[tiab] OR telehealth[tiab] OR "tele-health"[tiab] OR ehealth[tiab] OR "ehealth"[tiab] OR "Remote monitoring"[tiab] OR Tele-MAT[tiab] OR smartphone*[tiab] OR internet*[tiab] OR "internet-based"[tiab] OR "internet-delivered"[tiab] OR smartphone-based [tiab] OR smartwatch*[tiab] OR "apple watch"[ti] OR "i-watch"[ti] OR "i watch"[ti] OR "smartwatch"[ti] OR app-delivered[tiab] OR ict[tiab] OR telecare[tiab] OR "telecare devices"[tiab] OR "Mobile applications" [tiab] OR "distance medicine"[tiab] OR telemedical[tiab] OR teletherapy[tiab] OR "remote medicine"[tiab] OR "tele-health applications"[tiab] OR smart device*[tiab] OR app[tiab] OR virtual[tiab] OR "assistive technology"[tiab] OR "computerized assessment"[tiab] OR computer*[tiab] OR "electronic portable"[tiab] OR "Real-time simulator"[tiab] OR "assistive technology" [tiab] OR web-based[tiab] OR online[tiab] OR e-therapy OR "e therapy"[tiab] OR cellphone[ti] OR cellphone[ti] OR i-phone [ti] OR ios [ti] OR iphone[ti] OR ehealth[MESH] OR telemedicine [MESH] OR smartphone[MESH] )(monit*[tiab] OR adherence[tiab] OR compliance[tiab] or persistence[tiab] OR self-monitor*[tiab] OR "Drug Adherence"[MESH] OR "Medication Compliance" [MESH] OR "Drug Compliance"[MESH] OR "Treatment Adherence"[MESH] OR "Therapeutic Adherence"[MESH] )(“MS patients"[tiab]
OR "multiple sclerosis"[tiab] OR PPMS[tiab] OR RRMS[tiab] OR SPMS[tiab] OR "Disseminated Sclerosis"[tiab]).

Participant or population: Patients with Multiple Sclerosis.

Intervention: All digital health interventions such as following were included: smartphone, $\mathrm{m}$-health, smartwatch, etc.

Comparator: All other interventions in digital health will be included, studies with no control group will be included too.

Study designs to be included: Cohort and RCT studies were included.

Eligibility criteria: After systematic search of databases completed and duplicates studies were removed, we included: A) peer-reviewed published interventional studies B) that comprised of only digital health intervention/usage C) applied to medication adherence E) among patients with definite diagnosis of MS or as neurological samples where data related to MS patients could be extractedF) There were no language, ethnicity, age, sex and geographic limitation. We exclude articles of: 1.Non peer-reviewed articles and gray literature were excluded 2. Review articles, letter to editors, case reports, case series, meeting abstracts, animal studies, conference papers, study protocols and registered trials without report were excluded.

Information sources: The following databases were searched: PUBMED, EMBASE, SCOPUS, web of science.

Main outcome(s): Improving medication adherence.

Data management: All retrieved results were exported to the Endnote software and then Rayyan platform. Data such as country, gender, type of the disease and etc were extracted. all retrieved results were screened by at least two independent reviewers and any disagreement in screening or data extraction was solved by the third reviewer. 
Quality assessment / Risk of bias analysis: Quality assessment will be performed by JBI critical appraisal tool.

Strategy of data synthesis: Due to lack of meta-analysis in our study, the qualitative synthesis considered as a method of choice.

Subgroup analysis: None.

Sensitivity analysis: None.

Country(ies) involved: Iran.

Keywords: Digital health, medication adherence, Multiple sclerosis patients.

Contributions of each author:

Author 1 - Farhad Mahmoudi.

Email: farhad.mg116@yahoo.com

Author 2 - Mahtab Mokarram.

Email: mahtabmk95@gmail.com

Author 3 - Sadegh Sabouhi.

Email: s.sabouhi@live.com

Author 4 - Sara Hashemi.

Email: hashemi1979@gmail.com

Author 5 - Parastoo Saberi.

Email: parastoosaberi94@gmail.com

Author 6 - Hadi zamanian.

Email: hadi_zamanian@yahoo.com 